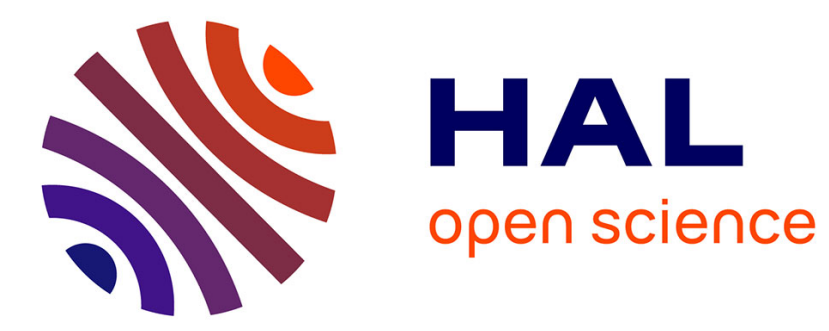

\title{
Edge-to-center plasma density ratios in two-dimensional plasma discharges
}

\author{
Romain Lucken, Vivien Croes, Trevor Lafleur, Jean-Luc Raimbault, A. \\ Bourdon, Pascal Chabert
}

\section{> To cite this version:}

Romain Lucken, Vivien Croes, Trevor Lafleur, Jean-Luc Raimbault, A. Bourdon, et al.. Edge-to-center plasma density ratios in two-dimensional plasma discharges. Plasma Sources Science and Technology, 2018, 27 (3), 10.1088/1361-6595/aaaf61 . hal-01860436

\section{HAL Id: hal-01860436 https://hal.science/hal-01860436}

Submitted on 23 Aug 2018

HAL is a multi-disciplinary open access archive for the deposit and dissemination of scientific research documents, whether they are published or not. The documents may come from teaching and research institutions in France or abroad, or from public or private research centers.
L'archive ouverte pluridisciplinaire HAL, est destinée au dépôt et à la diffusion de documents scientifiques de niveau recherche, publiés ou non, émanant des établissements d'enseignement et de recherche français ou étrangers, des laboratoires publics ou privés. 


\title{
Edge-to-center plasma density ratios in two-dimensional plasma discharges
}

\author{
R. Lucken $¥$ ॠ, V. Croes $^{1}$, T. Lafleur ${ }^{1}$ \\ J.-L. Raimbault ${ }^{1}$, A. Bourdon ${ }^{1}$, P. Chabert ${ }^{1}$ \\ ${ }^{1}$ LPP, CNRS, École polytechnique, UPMC Univ Paris 06, Univ. Paris-Sud, \\ Observatoire de Paris, Université Paris-Saclay, Sorbonne Universités, PSL Research \\ University, 91128 Palaiseau, France
}

October 2017

\begin{abstract}
.
Edge-to-center plasma density ratios - so-called $h$ factors - are important parameters for global models of plasma discharges as they are used to calculate the plasma losses at the reactor walls. There are well-established theories for $h$ factors in the one-dimensional case. The purpose of this paper is to establish $h$ factors in twodimensional (2D) systems, with guidance from a 2D particle-in-cell (PIC) simulation. We derive analytical solutions of a $2 \mathrm{D}$ fluid theory that includes the effect of ion inertia, but assumes a constant (independent of space) ion collision frequency (using an average ion velocity) across the discharge. Predicted $h$ factors from this $2 \mathrm{D}$ fluid theory have the same order of magnitude and the same trends as the PIC simulations when the average ion velocity used in the collision frequency is set equal to the ion thermal velocity. The best agreement is obtained when the average ion velocity varies with pressure (but remains independent of space), going from half the Bohm velocity at low pressure, to the thermal velocity at high pressure. The analysis also shows that a simple correction of the widely-used 1D heuristic formula may be proposed to accurately incorporate $2 \mathrm{D}$ effects.
\end{abstract}

Keywords: Pre-sheath drop, edge-to-center plasma density ratio, low-temperature plasma discharge

Submitted to: Plasma Sources Sci. Technol.

\section{Introduction}

Global models are handy tools to assess the scaling laws of a plasma discharge with respect to parameters such as the background pressure, the input power, and the geometry in numerous applications, for instance to predict the performances of plasma

‡ Email: romain.lucken@lpp.polytechnique.fr 
thrusters [1, 2, 3]. They are zero-dimensional (0D) models that yield an approximate solution of particle balance and power balance equations [4, 5]. It was shown in the past that these models are in good agreement with experiments [6], as well as with more detailed fluid or particle-in-cell (PIC) simulations [7].

In low temperature plasma discharges, the region near the wall is characterized by a sheath where quasi-neutrality is violated. According to the Bohm sheath criterion, ions must leave the bulk plasma and enter the sheath region at a velocity higher or equal to the Bohm velocity $u_{B}=\left(\frac{k T_{e}}{m_{i}}\right)^{1 / 2}, T_{e}$ being the electron temperature, $m_{i}$ the ion mass, and $k$ the Boltzmann constant. Since ionization is usually negligible in the sheath (at least in the low-pressure regimes considered in this paper), the ion flux reaching the wall is:

$$
\Gamma_{i}=n_{s} u_{B}
$$

where $n_{s}$ is the plasma density at the sheath edge. For global models however, the flux at the wall should be expressed as a function of the space-averaged density, which is not very different from the density at the center, noted $n_{0}$. It is then useful to introduce an $h$ factor, defined as

$$
h=n_{s} / n_{0}
$$

In the literature, this factor has been labeled $h_{L}$ for 1D Cartesian geometries or $h_{R}$ for 1D cylindrical geometries.

Several studies provided a good estimate of these factors over large pressure ranges, using various simplifications of the momentum conservation equations in one dimension (1D) [4, 5]. The transport in the higher pressure regime was first investigated by Schottky [8]. In this limit, the ion-neutral collision frequency only depends on the ion thermal velocity $\bar{v}_{i}=\left(\frac{8 k T_{i}}{\pi m_{i}}\right)^{1 / 2}$, where $T_{i}$ is the temperature of the ions. The lower pressure (collisionless) regime was studied by Tonks and Langmuir in the 1920's [9]. The intermediate pressure regime where the ion-neutral collision frequency becomes a function of the fluid velocity was described by Godyak [10] and more recently by Raimbault and Chabert [11]. Capitalizing on these works, Lee and Lieberman [12] provided a heuristic formula to estimate the one-dimensional $h$ factor in the entire pressure regimes, and this was revisited in Chabert and Braithwaite [5] to provide a simple formula for a 1D Cartesian system of size $l$ :

$$
h_{L}=0.86\left[3+0.5 \frac{l}{\lambda_{i}}+0.2 \frac{T_{i}}{T_{e}}\left(\frac{l}{\lambda_{i}}\right)^{2}\right]^{-0.5}
$$

where $\lambda_{i}$ is the ion mean free path. This formula has been shown to be in excellent agreement with 1D PIC simulations [7] of inductively coupled plasma (ICP) discharges, for which the electron temperature is fairly independent of space, and will be used as the $1 \mathrm{D}$ reference in this paper. It is useful to note that, to our knowledge, there is only one attempt of direct experimental validation of these simplified analytical solutions [13]. 
When trying to generalize the analysis to two or three dimensions, it may be useful to use a slightly different definition of the $h$ factor, starting from the global particle balance equation:

$$
n_{0} n_{g} K_{i z} V=\oiint_{S} \boldsymbol{\Gamma}_{i} \cdot \mathbf{d} \mathbf{S}
$$

where $n_{g}$ is the gas density. This equation says that the production of electron-ion pairs by ionization ( $K_{i z}$ is the ionization rate) in a plasma volume $V$ has to be balanced by the integral of the ion flux reaching the surface $S$ that encapsulates the plasma. Then, if the integral of the flux is written $h n_{0} u_{B} S$, the $h$ factor becomes:

$$
h=\frac{\oiint_{S} \boldsymbol{\Gamma}_{i} \cdot \mathbf{d} \mathbf{S}}{n_{0} u_{B} S}
$$

We will use this definition in this paper. We note that in this widely-used definition of the particle balance the following assumptions have been made:

- $n_{0}$ in the left-hand side (LHS) of Equation 4 is the maximum plasma density in the center of the discharge while it should rather be the average plasma density.

- Electrons and ions are isothermal.

- The dimension of the sheath is small compared to the reactor size.

In this paper we will discuss $h$ factors in 2D ICP discharges with Cartesian coordinates. In section 2, we describe the fluid theory of a non-magnetized plasma discharge for a two-dimensional (2D) Cartesian discharge that will be used as a theoretical support. We have used the work of Kino and Shaw [14] as a starting point for our calculation. In section 3, a description of the 2D PIC simulation used to verify the theory is provided. A wide range of pressure is investigated, from 0.3 to $100 \mathrm{mTorr}$, as well as several aspect ratios. Section 4 compares simulation results with the transport model for electron temperature and $h$ factors. Section 5 explores improvements of the theory and proposes a modified heuristic formula to incorporate $2 \mathrm{D}$ effects. Our conclusions are given in the last section.

\section{Theoretical approach of unmagnetized plasma transport in $2 \mathrm{D}$}

\subsection{System definition}

The system of interest is a rectangular plasma discharge of dimensions $l_{x}$ and $l_{y}$, as shown in Figure 1. The plasma is assumed to be sustained by an external power source that does not need to be specified. The system is assumed to be symmetrical with respect to $(O x)$ and $(O y)$ axis. In this configuration, from Equation 5 , the $h$ factor of the discharge is the following:

$$
h_{2 D}=\frac{2}{n_{0} u_{B}\left(l_{x}+l_{y}\right)}\left[\int_{0}^{l_{x} / 2} \Gamma_{y}\left(x, l_{y} / 2\right) d x+\int_{0}^{l_{y} / 2} \Gamma_{x}\left(l_{x} / 2, y\right) d y\right]
$$






Figure 1: Geometrical configuration of the plasma discharge.

The center of the system of coordinates is taken at the center of the discharge. By symmetry, the fluid velocity is null in the discharge center, and so is the density gradient. The plasma density drops nearly to 0 at the walls, so the simplest boundary condition, known as the Schottky criterion, is the following:

$$
n(x, y)=0 \text { when } x= \pm l_{x} / 2, \text { or } y= \pm l_{y} / 2
$$

This condition is an acceptable approximation to calculate the electron temperature but is not consistent since the flux is finite at the boundary. In addition, it cannot be used directly to evaluate $h$ factors. A better boundary condition is therefore to use the Bohm flux at the boundaries.

\subsection{General fluid equations}

The approach used here aims at generalizing the model investigated in [14] to all regimes of pressures, with the assumption that ion-neutral collisions and ionization can be accounted for with constant (i.e. independent of fluid velocity or position) collision frequencies. We will extensively discuss the consequence of this assumption later in the text since it is known to be invalid in the intermediate pressure regime. The momentum conservation equations for ions and electrons are:

$$
\left\{\begin{aligned}
m_{i} \mathbf{u} \nabla \mathbf{u} & =e \mathbf{E}-k T_{i} \frac{\nabla n}{n}-\left(\nu_{i z}+\nu_{i}\right) m_{i} \mathbf{u} \\
0 & =-e \mathbf{E}-k T_{e} \frac{\nabla n}{n}
\end{aligned}\right.
$$

where ion inertia is included and, as in previous models, Boltzmann electrons are used. The continuity equation is:

$$
\nabla \cdot(n \mathbf{u})=\nu_{i z} n
$$


Expanding these equations on both axis $(O x)$ and $(O y)$ :

$$
\left\{\begin{aligned}
\left(u_{x} \partial_{x}+u_{y} \partial_{y}\right) u_{x} & =-u_{B}^{2} \frac{\partial_{x} n}{n}-\left(\nu_{i z}+\nu_{i}\right) u_{x} \\
\left(u_{x} \partial_{x}+u_{y} \partial_{y}\right) u_{y} & =-u_{B}^{2} \frac{\partial_{y} n}{n}-\left(\nu_{i z}+\nu_{i}\right) u_{y} \\
\partial_{x}\left(n u_{x}\right)+\partial_{y}\left(n u_{y}\right) & =\nu_{i z} n
\end{aligned}\right.
$$

where it was assumed that $T_{i} \ll T_{e}$. We look for solutions by separation of variables:

$$
n=n_{0} f(x) g(y) \quad ; \quad \mathbf{u}=\left[\begin{array}{l}
u_{x}(x) \\
u_{y}(y)
\end{array}\right]
$$

where $f$ and $g$ are arbitrary even functions of $x$ and $y$ respectively and $n_{0}$ is the plasma density in the center of the discharge. Normalization implies that $f(0)=g(0)=1$, and by symmetry, $u_{x}(0)=u_{y}(0)=0$. The continuity equation becomes:

$$
\underbrace{u_{x} \frac{f^{\prime}}{f}+u_{x}^{\prime}}_{=F(x)}+\underbrace{u_{y} \frac{g^{\prime}}{g}+u_{y}^{\prime}}_{=G(y)}=\nu_{i z}
$$

The sum of $F(x)$ and $G(y)$ is constant, which implies that both of these functions are constants which will be called $\nu_{x}$ and $\nu_{y}$ in the following. Thus, the continuity equation can be separated in two parts:

$$
\begin{aligned}
& u_{x} \frac{f^{\prime}}{f}+u_{x}^{\prime}=\nu_{x} \\
& u_{y} \frac{g^{\prime}}{g}+u_{y}^{\prime}=\nu_{y}
\end{aligned}
$$

with

$$
\nu_{x}+\nu_{y}=\nu_{i z}
$$

The momentum conservation equations for each coordinates are:

$$
\begin{aligned}
& u_{x} u_{x}^{\prime}=-u_{B}^{2} \frac{f^{\prime}}{f}-\left(\nu_{i z}+\nu_{i}\right) u_{x} \\
& u_{y} u_{y}^{\prime}=-u_{B}^{2} \frac{g^{\prime}}{g}-\left(\nu_{i z}+\nu_{i}\right) u_{y}
\end{aligned}
$$

We can substitute $f^{\prime} / f$ in Equation 16 using $f^{\prime} / f=\left(\nu_{x}-u_{x}^{\prime}\right) / u_{x}$, leading to

$$
u_{x}^{\prime}+\frac{u_{B}^{2}}{u_{x}^{2}}\left(\nu_{x}-u_{x}^{\prime}\right)+\nu_{i z}+\nu_{i}=0 \Leftrightarrow \frac{u_{x}^{2}-u_{B}^{2}}{\nu_{x} u_{B}^{2}+\left(\nu_{i z}+\nu_{i}\right) u_{x}^{2}} d u_{x}=-d x
$$

such that, after integrating this equation and its equivalent in the $y$ direction, we find:

$$
\begin{aligned}
& x=\frac{\nu_{i z}+\nu_{i}+\nu_{x}}{\nu_{x}^{1 / 2}\left(\nu_{i}+\nu_{i z}\right)^{3 / 2}} u_{B} \arctan \left[\left(\frac{\nu_{i z}+\nu_{i}}{\nu_{x}}\right)^{1 / 2} \frac{u_{x}}{u_{B}}\right]-\frac{u_{x}}{\nu_{i z}+\nu_{i}} \\
& y=\frac{\nu_{i z}+\nu_{i}+\nu_{y}}{\nu_{y}^{1 / 2}\left(\nu_{i}+\nu_{i z}\right)^{3 / 2}} u_{B} \arctan \left[\left(\frac{\nu_{i z}+\nu_{i}}{\nu_{y}}\right)^{1 / 2} \frac{u_{y}}{u_{B}}\right]-\frac{u_{y}}{\nu_{i z}+\nu_{i}}
\end{aligned}
$$


Since the ions leave the plasma at the Bohm speed and we are in the limit $l_{x}, l_{y} \gg s$, it follows that:

$$
\begin{aligned}
& l_{x}=\frac{2 u_{B}}{\nu_{i z}+\nu_{i}}\left[\frac{\nu_{i}+\nu_{i z}+\nu_{x}}{\sqrt{\nu_{x}\left(\nu_{i}+\nu_{i z}\right)}} \arctan \left(\frac{\nu_{i z}+\nu_{i}}{\nu_{x}}\right)^{1 / 2}-1\right] \\
& l_{y}=\frac{2 u_{B}}{\nu_{i z}+\nu_{i}}\left[\frac{\nu_{i}+\nu_{i z}+\nu_{y}}{\sqrt{\nu_{y}\left(\nu_{i}+\nu_{i z}\right)}} \arctan \left(\frac{\nu_{i z}+\nu_{i}}{\nu_{y}}\right)^{1 / 2}-1\right]
\end{aligned}
$$

Equations 20 and 21, combined with Equation 15, will set the electron temperature, and the variables $\nu_{x}$ and $\nu_{y}$.

As detailed in the Appendix, rearranging the momentum conservation equations and the continuity equations leads after integration to:

$$
\begin{aligned}
& f(x)=\left[1+\frac{u_{x}(x)^{2}}{u_{B}^{2}} \frac{\nu_{i z}+\nu_{i}}{\nu_{x}}\right]^{-\frac{1}{2}\left(1+\frac{\nu_{x}}{\nu_{i z}+\nu_{i}}\right)} \\
& g(y)=\left[1+\frac{u_{y}(y)^{2}}{u_{B}^{2}} \frac{\nu_{i z}+\nu_{i}}{\nu_{y}}\right]^{-\frac{1}{2}\left(1+\frac{\nu_{y}}{\nu_{i z}+\nu_{i}}\right)}
\end{aligned}
$$

where $u_{x}$ and $u_{y}$ are given by the implicit form of Equations 18 and 19 . At the boundaries, $x=l_{x} / 2$ and $y=l_{y} / 2$, the normalized densities in the $(O x)$ and $(O y)$ directions $-f_{s}=f\left(l_{x} / 2\right)$ and $g_{s}=g\left(l_{y} / 2\right)$ respectively - are given by the Bohm criteria $u_{x}\left(l_{x} / 2\right)=u_{B}$ and $u_{y}\left(l_{y} / 2\right)=u_{B}$ :

$$
\begin{aligned}
& f_{s}=\left(1+\frac{\nu_{i z}+\nu_{i}}{\nu_{x}}\right)^{-\frac{1}{2}\left(1+\frac{\nu_{x}}{\nu_{i z}+\nu_{i}}\right)} \\
& g_{s}=\left(1+\frac{\nu_{i z}+\nu_{i}}{\nu_{y}}\right)^{-\frac{1}{2}\left(1+\frac{\nu_{y}}{\nu_{i z}+\nu_{i}}\right)}
\end{aligned}
$$

It becomes clear that the edge-to-center plasma density ratio depends on the location where it is evaluated. For example:

$$
\left\{\begin{array}{l}
h_{L}(y)=\frac{n\left(l_{x} / 2, y\right)}{n_{0}}=f_{s} g(y) \\
h_{L}(x)=\frac{n\left(x, l_{y} / 2\right)}{n_{0}}=g_{s} f(x)
\end{array}\right.
$$

Using Equation 6 and the fact that

$$
\left\{\begin{array}{l}
\Gamma_{y}\left(x, l_{y} / 2\right)=n\left(x, l_{y} / 2\right) u_{B} \\
\Gamma_{x}\left(l_{x} / 2, y\right)=n\left(l_{x} / 2, y\right) u_{B}
\end{array}\right.
$$

we find:

$$
h_{2 D}=\frac{2}{l_{x}+l_{y}}\left[\int_{0}^{l_{x} / 2} h_{L}(x) d x+\int_{0}^{l_{y} / 2} h_{L}(y) d y\right]=\frac{l_{x} g_{s} \beta_{x}+l_{y} f_{s} \beta_{y}}{l_{x}+l_{y}}
$$


where $\beta_{x}$ and $\beta_{y}$ are the mean profiles in $(O x)$ and $(O y)$ directions respectively. They can be calculated easily by integrating Equations 13 and 14 .

$$
\begin{aligned}
& \beta_{x}=\frac{2}{l_{x}} \int_{0}^{l_{x} / 2} f(x) d x=\frac{2 u_{B}}{l_{x} \nu_{x}} f_{s} \\
& \beta_{y}=\frac{2}{l_{y}} \int_{0}^{l_{y} / 2} g(y) d y=\frac{2 u_{B}}{l_{y} \nu_{y}} g_{s}
\end{aligned}
$$

Finally,

$$
h_{2 D}=\frac{\beta_{x} \beta_{y} l_{x} l_{y} \nu_{i z}}{2 u_{B}\left(l_{x}+l_{y}\right)}
$$

which is consistent with the global particle balance of Equation 4 .

\subsection{Study of the limit cases}

2.3.1. High pressure Since in the high pressure case:

$$
\nu_{i} \gg \nu_{i z}>\nu_{x}, \nu_{y}
$$

Equation 20 may be simplified since $\arctan \left(\frac{\nu_{i z}+\nu_{i}}{\nu_{x}}\right)^{1 / 2} \approx \frac{\pi}{2}$, leading to

$$
l_{x}=\frac{\pi u_{B}}{\sqrt{\nu_{x} \nu_{i}}}
$$

Using the same approximation along $(O y)$ gives:

$$
\nu_{x, y}=\frac{\pi^{2} u_{B}^{2}}{\nu_{i} l_{x, y}^{2}}
$$

such that the condition $\nu_{x}+\nu_{y}=\nu_{i z}$ becomes:

$$
\frac{\pi^{2} u_{B}^{2}}{\nu_{i}}\left(\frac{1}{l_{x}^{2}}+\frac{1}{l_{y}^{2}}\right)=\nu_{i z} \quad \text { or } \quad \frac{\pi^{2}}{l_{x}^{2}}+\frac{\pi^{2}}{l_{y}^{2}}=\frac{\nu_{i z}}{D_{a}}
$$

Taking this limit in Equations 24 and 25 yields:

$$
f_{s}=\sqrt{\frac{\nu_{x}}{\nu_{i}}}=\frac{\pi u_{B}}{\nu_{i} l_{x}} g_{s}=\sqrt{\frac{\nu_{y}}{\nu_{i}}}=\frac{\pi u_{B}}{\nu_{i} l_{y}}
$$

such that the two-dimensional $h$ factor is, from Equation 28 ;

$$
h_{2 D}=\frac{2 u_{B}}{\nu_{i}\left(l_{x}+l_{y}\right)}\left(\frac{l_{y}}{l_{x}}+\frac{l_{x}}{l_{y}}\right)
$$

As shown below, these approximate solutions are consistent with the more classical solutions of the Helmholtz equation that describes the high pressure regime for a $2 \mathrm{D}$ Cartesian system [4]:

$$
n=n_{0} \cos \left(k_{x} x\right) \cos \left(k_{y} y\right), \text { with } k_{x}^{2}+k_{y}^{2}=\frac{\nu_{i z} \nu_{i}}{u_{B}^{2}}
$$


Using Schottky boundary conditions ( $n=0$ at the plasma edge) and assuming that the sheath thickness $s$ is much smaller than the system size, $s \ll l_{x} / 2$, the following conditions are found:

$$
k_{x}=\frac{\pi}{l_{x}} \quad ; \quad k_{y}=\frac{\pi}{l_{y}}
$$

which yields exactly the same equation as Equation 35 for the electron temperature.

2.3.2. Low pressure At low pressure, the ion-neutral collision frequency is negligible, such that, from Equation 24:

$$
f_{s}=\left(1+\frac{\nu_{i z}}{\nu_{x}}\right)^{-\frac{1}{2}\left(1+\frac{\nu_{x}}{\nu_{i z}}\right)}
$$

For a $1 \mathrm{D}$ discharge, $l_{y}$ is infinite, $\nu_{y}=0$, and $\nu_{x}=\nu_{i z}$. Thus, $f_{s}=2^{-1}=0.5$, which is the classical result of the low-pressure limit [5], when ionization is included in the momentum balance equation. For a square, $\nu_{x}=\nu_{y}=\nu_{i z} / 2$, and $f_{s}=3^{-3 / 4} \approx 0.44$. This shows that the $h$ factor shows a clear dependance on the aspect ratio of the discharge.

2.3.3. Large aspect ratios Equation 20 implies that when $l_{x}$ becomes very large $\nu_{x}$ becomes very small, such that the following approximation may be used:

$$
l_{x} \nu_{x} \approx \pi u_{B}\left(\frac{\nu_{x}}{\nu_{i}+\nu_{i z}}\right)^{1 / 2}
$$

In addition, taking $\nu_{x}$ very small in Equation 24 leads to $f_{s}=\left(\frac{\nu_{x}}{\nu_{i}+\nu_{i z}}\right)^{1 / 2}$. The average of the normalized density profile is then simply $\beta_{x}=2 / \pi$, which is the same as the high pressure case. It is well known that the parameter that sets the shape of the density profile is the product of the pressure by the length. It therefore makes sense that increasing the length in one direction leads to the same effect on $\beta_{x}$ as increasing the pressure.

\subsection{General solutions}

The analytical formulae derived in section 2.2 can be used to deduce the $h$ factor once the electron temperature has been determined. In this section we explain the procedure used to solve Equations 20, 21, and 15 for the variables $T_{e}, \nu_{x}$ and $\nu_{y}$. We introduce the variables $\theta$ and $\phi$, defined as

$$
\begin{aligned}
\sin ^{2} \phi & =\frac{\nu_{i z}}{\nu_{i z}+\nu_{i}} \\
\tan ^{2} \theta & =\frac{\nu_{y}}{\nu_{x}}
\end{aligned}
$$


so that Equations 20 and 21 take the more compact form:

$$
\begin{aligned}
& \frac{l_{x} \nu_{i}}{2 u_{B}}=\cos ^{2} \phi G(\sin \phi \cos \theta) \\
& \frac{l_{y} \nu_{i}}{2 u_{B}}=\cos ^{2} \phi G(\sin \phi \sin \theta)
\end{aligned}
$$

with $G(x)=(1 / x+x) \arctan \left(\frac{1}{x}\right)-1$. Arranging those two equations, we obtain:

$$
G(\sin \theta \sin \phi)-\frac{l_{y}}{l_{x}} G(\cos \theta \sin \phi)=0
$$

which can be solved numerically to get $\theta$ as a function of $l_{y} / l_{x}$ and $\phi$. The relation between the ionization frequency, $\nu_{i z}=n_{g} K_{i z}$, and the electron temperature is gas dependent. In this paper we consider argon, and the ionization rate factor $K_{i z}$ is taken to be [4]:

$$
K_{i z}=2.34 \times 10^{-14} T_{e}^{0.59} e^{-17.44 / T_{e}}
$$

where $T_{e}$ is expressed in volts. In the first set of results, the ion-neutral collision frequency is taken to be

$$
\nu_{i}=\frac{\bar{v}_{i}}{\lambda_{i}}
$$

The gas pressure $p$, and the gas temperature $T_{g}$ are input parameters of the model. The gas density $n_{g}$ and the ion mean free path $\lambda_{i}$ are obtained from the following formulae:

$$
p[\mathrm{mTorr}]=\frac{n_{g} k T_{g}}{0.133}=\frac{k T_{g}}{0.133 \sigma_{i} \lambda_{i}}
$$

where $\sigma_{i}$ is the average cross section for ion-neutral collisions, assumed constant and equal to $\sigma_{i}=1.0 \times 10^{-18} \mathrm{~m}^{2}$, as done in references [7, 15].

Note that the spatially constant ion-neutral collision frequency proposed above should be valid at higher pressure (typically $p>100$ mTorr for systems of a few centimeters of size), and since $\nu_{i}$ becomes negligible at low pressure compared to $\nu_{i z}$, the low pressure regime is also described properly. An improved expression for the ionneutral collision frequency will be proposed in the section 5 in order to better describe the intermediate pressure regime.

At this point, $\phi$ is now a given function of the electron temperature, while the pressure and the size of the discharge are parameters. Thus, Equation 44 becomes an equation for the electron temperature which can be solved numerically. This provides solutions for $\nu_{x}$ and $\nu_{y}$ that can be used in all the analytical expressions of section 2.2. These numerical solutions are compared to PIC simulation results in section 4. Approximate solutions of the model are also possible and detailed in the Appendix, as well as in [16]. 


\section{2D Particle-In-Cell (PIC) simulations}

\subsection{Description of the LPPic2D code}

We have used a 2D parallelized Particle-In-Cell (PIC) code called LPPic2D, developed in-house for the simulation of low temperature plasmas, and described in details in [17. This code uses a uniform structured mesh, constant time steps, and uses a Monte-Carlo collision (MCC) routine to account for the collisions between the charged species and the neutral gas. Momentum transfer collision cross sections coming from Phelps [18] for ion-neutral reactions and Biagi [19] for electron-neutral reactions are implemented. Electron-neutral reactions include elastic collisions, first ionization, and several excitation reactions. Three levels of excitation are resolved for argon. There are two main reactions for ion-neutral collisions which are elastic scattering and backscattering. The code is electrostatic, so only Poisson's equation is solved at each time step, and the magnetic field induced by the motion of the charged particles is neglected. At steady-state, the simulation matches the cell size and time step criteria described in [20]. Moreover, LPPic2D has been benchmarked using the reference cases published by Turner et al. [21].

The main assumptions of the model are the following:

- The neutral gas density is assumed to be much larger than those of charged species. Therefore, it is assumed to remain constant and uniform over the simulation run neutral density and temperature are input parameters of the simulation

- Short-range Coulomb collisions between charged species are neglected.

- The dynamics of excited species is not taken into account. Excitation reactions are treated as energy losses.

- Walls are conducting and grounded so they are not charged by impinging particles.

- Finally, secondary electron emission (SEE) is neglected.

A new module was implemented to model an ICP generation. This method was already described by Lafleur and Chabert [7] for 1D simulations of an ICP and extended here in 2D. The heating electric field is uniform over the simulation domain and it is directed along the direction perpendicular to the simulation plane. The absorbed power by the plasma, $P_{a b s}$, is set as an input parameter. We then impose the following electric field,

$$
\mathbf{E}=E_{0} \cos (\omega t) \mathbf{e}_{\mathbf{z}}
$$

and the amplitude $E_{0}$ is updated at the end of each radio-frequency $(\mathrm{RF})$ cycle of period $T_{R F}$ using

$$
E_{0}=\frac{P_{a b s} T_{R F} l_{x} l_{y}}{\int_{t}^{t+T_{R F}} \iint j_{z} \cos (\omega t) d x d y d t}
$$

in order to keep the power absorbed by the plasma constant, where $j_{z}$ is the electron current density along $(O z)$. 


\begin{tabular}{lccr}
\hline Discharge size in $(O x)$ direction & $l_{x}$ & $0.75-7.5$ & $\mathrm{~cm}$ \\
Discharge size in $(O y)$ direction & $l_{y}$ & 3 & $\mathrm{~cm}$ \\
Neutral gas pressure & $p_{n}$ & $0.3-100$ & $\mathrm{mTorr}$ \\
Neutral gas density & $n_{g}$ & $9.6 \times 10^{18}-3.2 \times 10^{21}$ & $\mathrm{~m}^{-3}$ \\
Ion mean free path (Ar) & $\lambda_{i}$ & $0.031-10$ & $\mathrm{~cm}$ \\
Neutral gas temperature & $T_{g}$ & 300 & $\mathrm{~K}$ \\
Power absorbed by the plasma & $P_{a b s}$ & 19.1 & $\mathrm{~kW} / \mathrm{m}^{3}$ \\
Excitation frequency & $f_{R F}$ & 13.56 & $\mathrm{MHz}$ \\
Number of cells & $N_{c e l l}$ & $40000-400000$ & \\
Time step & $d t$ & $1 \times 10^{-11}-3 \times 10^{-11}$ & $\mathrm{~s}$ \\
Number of CPUs & $n_{\text {proc }}$ & $200-400$ & \\
\hline
\end{tabular}

Table 1: Typical simulation parameters.

The electric field in the simulation plane is derived from the potential, which is the solution of Poisson's equation computed at each time step. The main simulation parameters that were used here are summarized in Table 1.

Figure 2 shows the plasma density obtained in steady-state by the 2D PIC simulation for an argon ICP discharge at $10 \mathrm{mTorr}$ and $19 \mathrm{~kW} / \mathrm{m}^{3}$. The sheath region, where the electron density drops to nearly zero, while ion density decreases smoothly, is clearly visible in Figure 2(b) and is small compared to the bulk plasma region.

\subsection{Variable separation}

Examining the PIC simulations, it was verified whether or not looking for analytical solutions with separated variables is reasonable. In this prospect, original PIC simulation results for plasma density, $n(x, y)$ were compared with the convoluted density $n_{\text {conv }}(x, y)=\frac{n(x, 0) n(0, y)}{n_{0}}$, where $n_{0}$ is the plasma density in the center of the discharge. The model detailed in this paper applies only in the bulk and pre-sheath plasma regions so variable separation in the sheath region is not investigated. The density profiles at the sheath edge - where the discrepancy between the convoluted density and the real one should be the most critical - are plotted in Figure 3 for runs at 1 mTorr, 10 mTorr, and $100 \mathrm{mTorr}$. The agreement between the real solution and the convoluted one is within the noise (about $5 \times 10^{14} \mathrm{~m}^{-3}$ ) at $100 \mathrm{mTorr}$, and it is still very satifsactory at the lower pressures.

\section{Comparison between the fluid theory and the PIC simulations}

In this section we compare the electron temperature and the $h$ factor using a constant ion-neutral collision frequency $\nu_{i}=\bar{v}_{i} / \lambda_{i}$ (Equation 48) in the fluid theory. A large range of pressure is investigated, from the collisionless regime to the higher pressure Schottky regime. We also investigate the effect of the aspect ratio. 


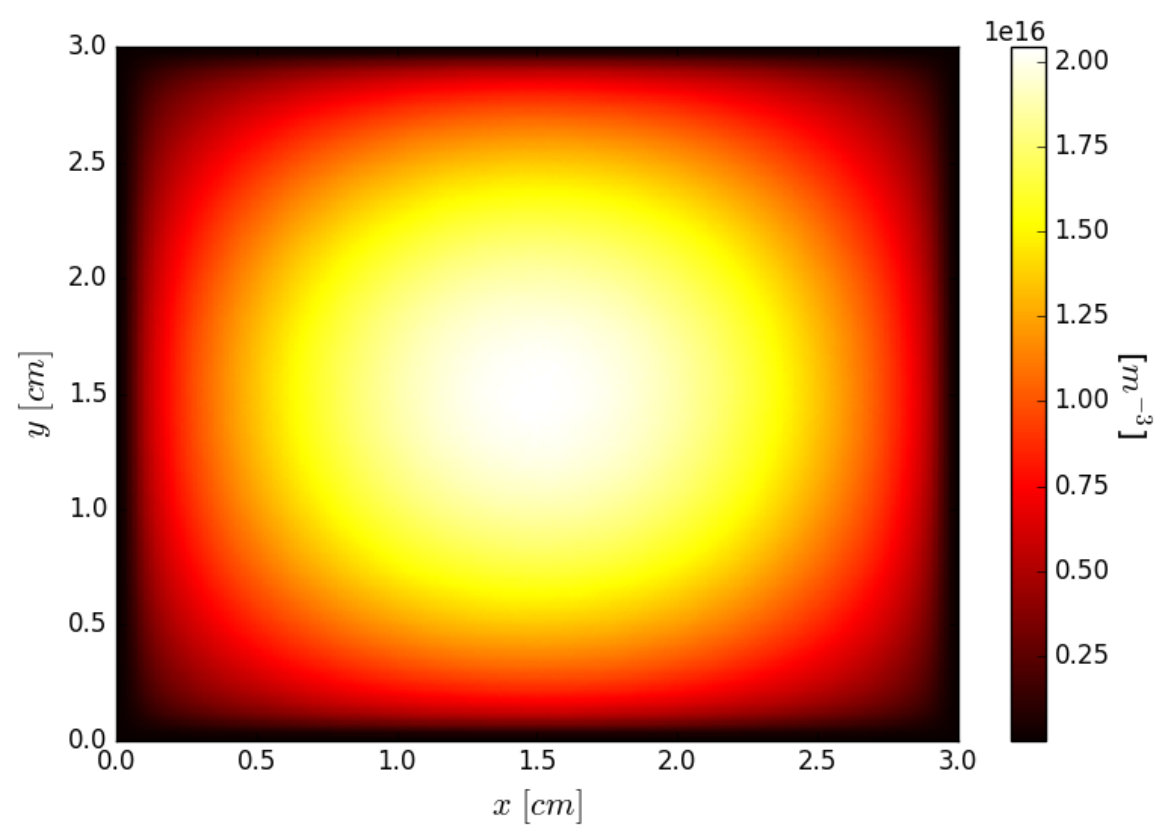

(a)

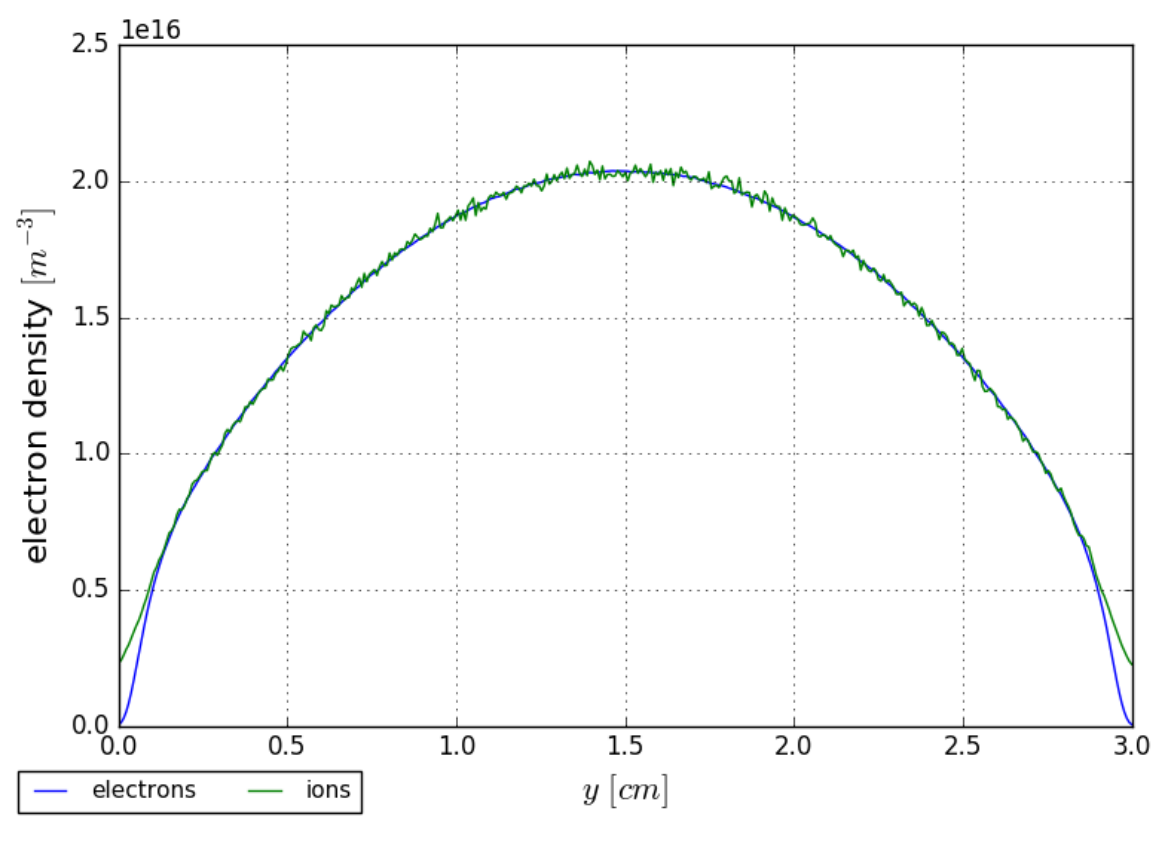

Figure 2: Electron and ion densities in steady-state for an argon ICP discharge at $10 \mathrm{mTorr}$ and $19 \mathrm{~kW} / \mathrm{m}^{3}$. (a) Electron density in color plot. (b) Densities of both species represented for $x=l_{x} / 2$.

\subsection{Electron temperature}

The variation of the electron temperature as a function of $l_{x} / \lambda_{i}$ is shown in Figure 4. The solid lines are the fluid theory solutions while the dots are the PIC simulation results. Two sets of data are presented. In the first one, $l_{x}=l_{y}=3 \mathrm{~cm}$ and the pressure is varied from $0.2 \mathrm{~m}$ Torr to 1.0 Torr in order to vary $l_{x} / \lambda_{i}$. In the second set of data, the $y$-direction size is kept constant at $l_{y}=3.0 \mathrm{~cm}$, the pressure is fixed at $p=10 \mathrm{~m}$ Torr, 


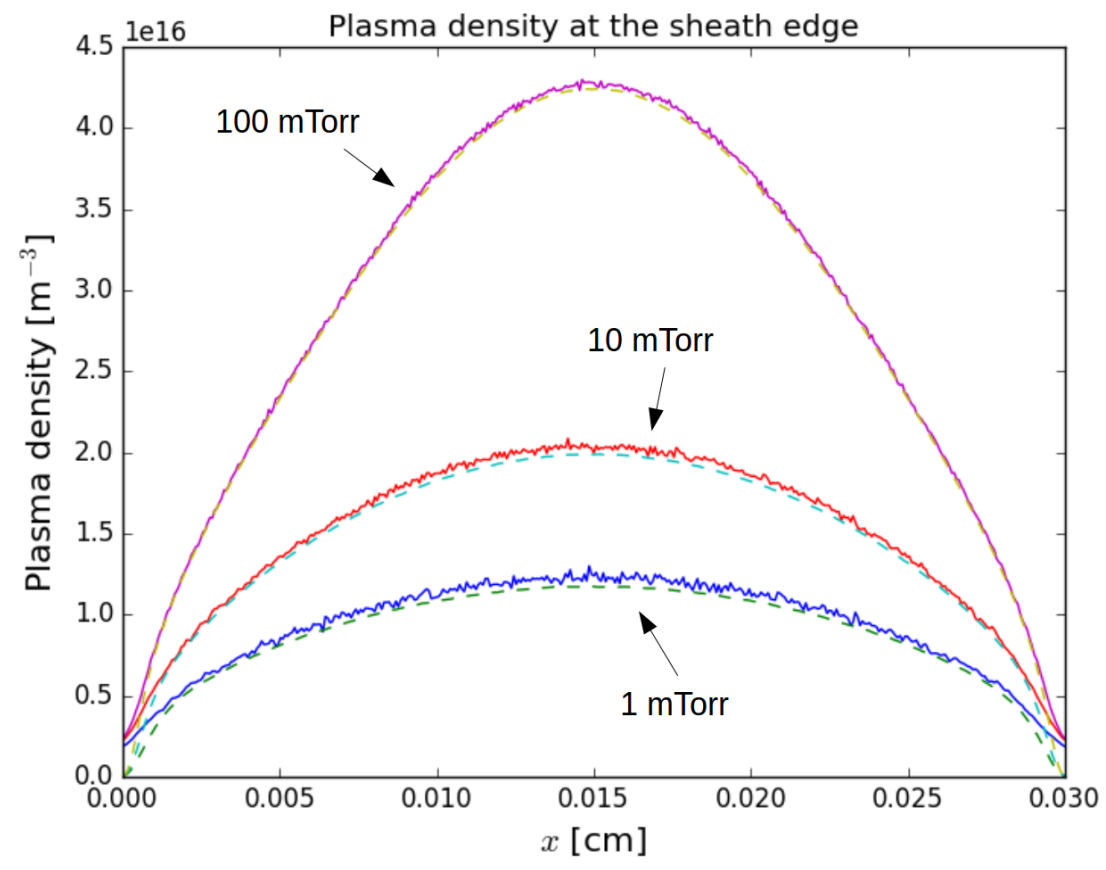

Figure 3: Ion density at the sheath edge for various values of pressure. Real profiles are plotted with solid lines and reconstructed profiles with dashed lines.

and $l_{x}$ is varied from $0.6 \mathrm{~mm}$ to $6 \mathrm{~m}$ in order to vary $l_{x} / \lambda_{i}$. The curves for the two sets of data cross at $l_{x} / \lambda_{i}=l_{y} / \lambda_{i}=10$, i.e. for a square discharge. Both curves show a decrease of the electron temperature with $l_{x} / \lambda_{i}$, but the decrease is faster when the pressure is varied than when $l_{x}$ is varied.

In both cases a very good agreement is found between the theory and the PIC simulations at low pressure. There is a significant disagreement between the theory and the PIC simulations at the higher pressures, mostly because the electron isothermal assumption breaks down. Indeed, in our PIC simulations, the RF heating electric field is independent of space but since the electron density is not, the power deposition is not uniform. At low pressure, the electron kinetic is largely non-local and consequently the electron temperature remains uniform even if the power absorption is not. At higher pressure, the electron temperature starts to peak in the center, where the absorbed power is the larger, and consequently the assumption of uniform electron temperature is violated.

\section{2. $h$ factor}

The global $h$ factors, labeled $h_{2 D}$, are computed from PIC simulations and compared to the predictions of the theory described in section 2. In the PIC simulations, the $h$ factors are calculated from the ion current collected at the system boundaries, the central electron density, and the Bohm velocity (computed using the mean electron temperature across the discharge), using the definition provided in Equation 5 . Figure 5 shows $h_{2 D}$ 


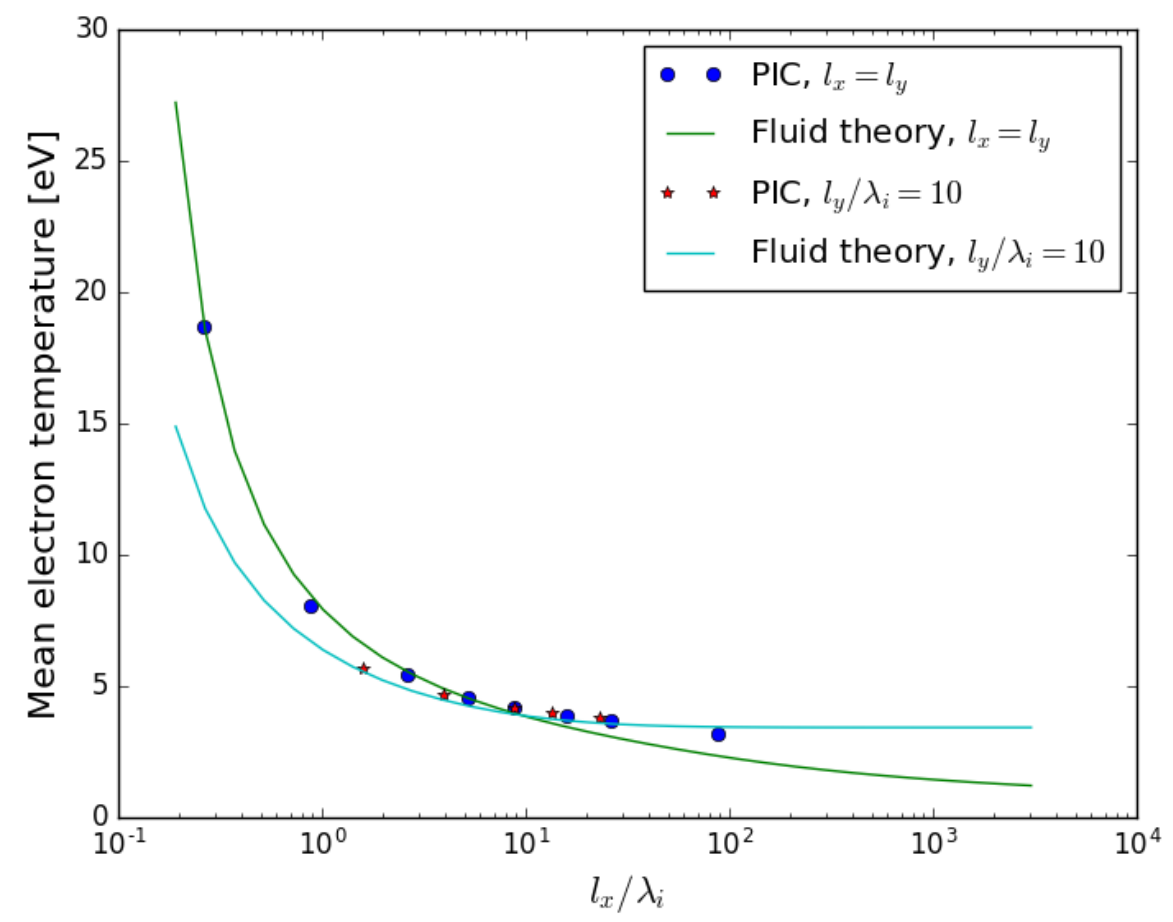

Figure 4: Electron temperature for various pressures and aspect ratios, with $l_{y}=3 \mathrm{~cm}$. The solid lines are the fluid theory solutions while the dots are the PIC simulation results.

as a function of $l_{x} / \lambda_{i}$ when the pressure is varied, while figure 6 shows $h_{2 D}$ as a function of $l_{x} / \lambda_{i}$ when $l_{x}$ is varied. The trends are captured by the theory, but the agreement between the theory and the PIC simulation is not very good, in particular when the aspect ratio is varied (Figure 6). The theory curve is also significantly higher than the PIC results in the intermediate pressure regime in Figure 5. As will be discussed in the next section, the theory can be improved by using a more appropriate expression for the ion-neutral collision frequency.

\section{Improved theory and heuristic formula}

\subsection{The intermediate pressure regime}

The so-called intermediate pressure regime corresponds to a common situation in industrial plasma applications where the characteristic size of the system is a few times the ion mean free path $\left(1<l / \lambda_{i}<100\right)$. Godyak [10] showed that the ion-neutral collision frequency should, in this case, depend on the local fluid velocity of the ions. This leads to non-linear transport equations, that are difficult to solve analytically, even in 1D. The fluid model used in this paper was already investigated by Sternberg and Godyak 22] for intermediate pressure discharges in 2D cylindrical coordinates, but no verification of the model was provided, either with experiments or simulations. 


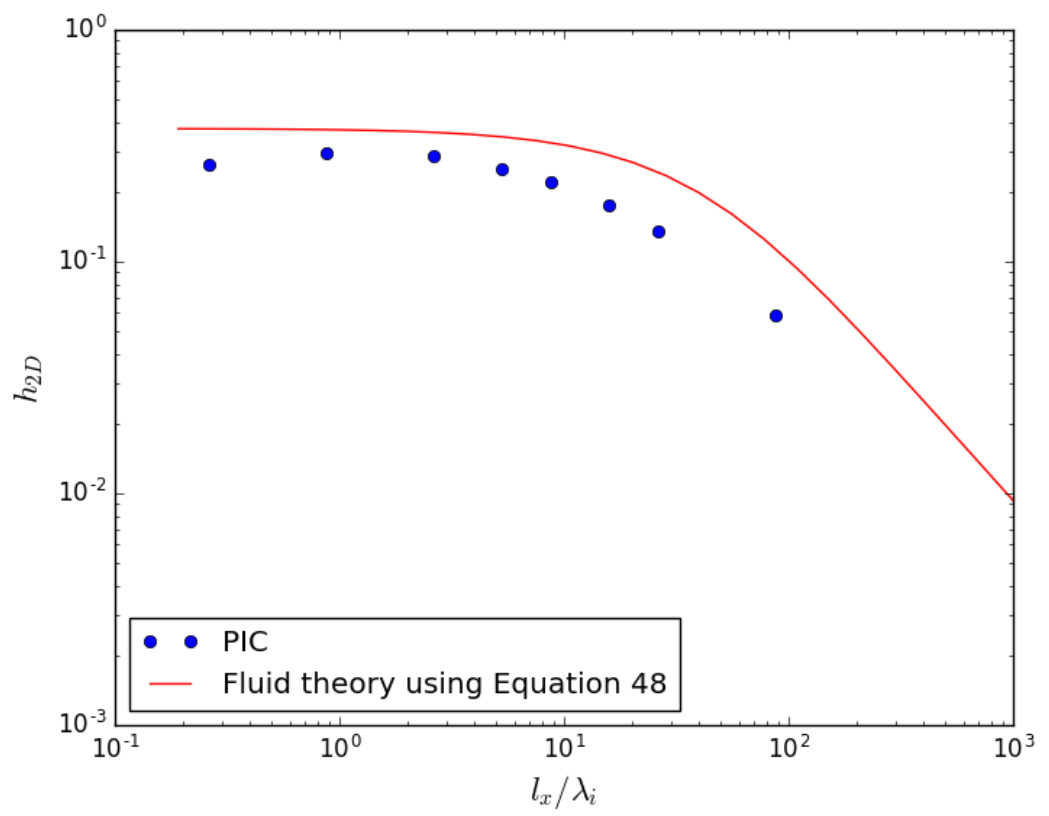

Figure 5: $h_{2 D}$ factors from the fluid theory (solid line) compared with PIC simulation results (dots) for various values of pressure. $l_{x}=l_{y}=3 \mathrm{~cm}, p$ from $0.2 \mathrm{~m}$ Torr to 1.0 Torr.

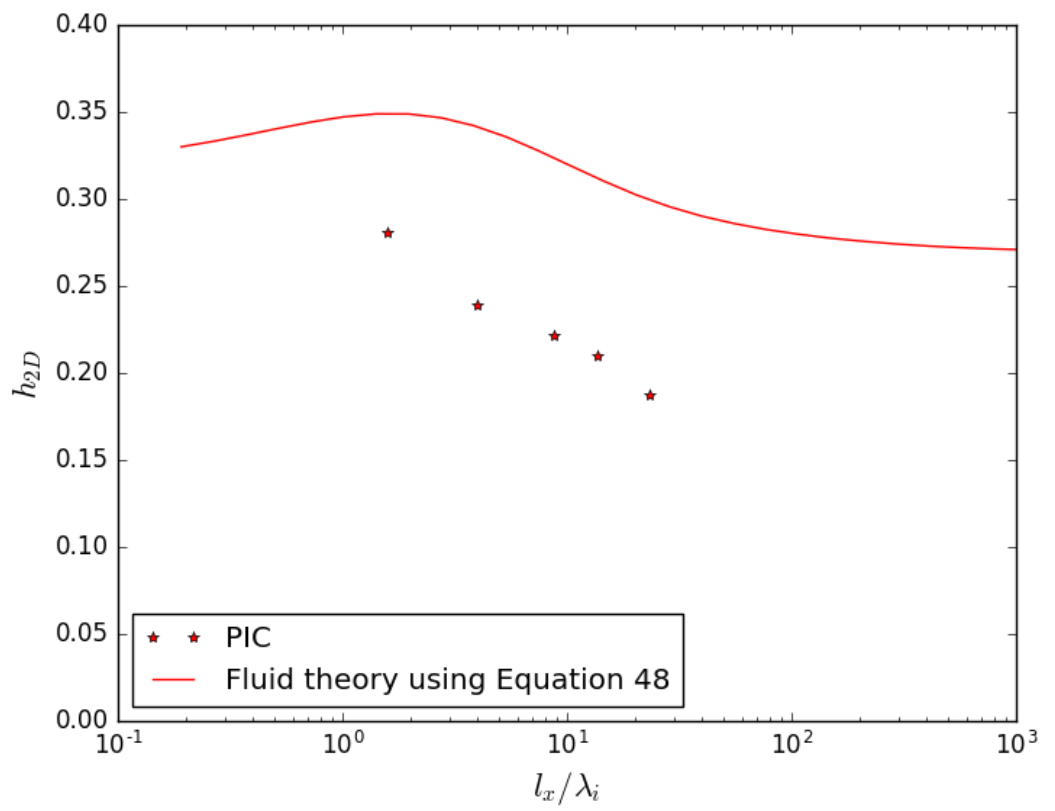

Figure 6: $h_{2 D}$ factors from the fluid theory (solid line) compared with PIC simulation results (dots) for various dimensions in $(O x)$ direction. $l_{y}=3.0 \mathrm{~cm}, p=10 \mathrm{~m}$ Torr, $l_{x}$ from $0.6 \mathrm{~mm}$ to $6 \mathrm{~m}$. 
In the fluid model used so far in this paper, the ion-neutral collision frequency was $\nu_{i}=\bar{v}_{i} / \lambda_{i}$ (Equation 48) which is a good approximation at high pressure. At very low pressure, the ion-collision frequency goes to zero and therefore the very low-pressure regime (typically $l<\lambda_{i}$ ) was also well described by the model even when Equation 48 was used. Nevertheless, as seen in Figure 5, the model has to be improved by a better choice of the ion-neutral collision frequency for low and intermediate pressures. We propose to use the form

$$
\nu_{i}=v_{a v} / \lambda_{i}
$$

where $v_{a v}$ is a typical average velocity of the ions, independent of the position in the discharge, but not independent of the pressure. For all pressure regimes, the ions start at zero fluid velocity in the center and exit the plasma at the Bohm speed. At low pressure, the weakly collisional ions are quickly accelerated and the fluid velocity becomes rapidly higher than the thermal velocity. In this situation, the average ion velocity $v_{a v}$ should be close to half the Bohm velocity. At high pressure, the ions drift slowly and the fluid velocity is below the thermal velocity in most of the discharge. Based on these observations, the following formula was proposed for pressure-dependent average velocity of the ions:

$$
v_{a v}=\bar{v}_{i}+b(\alpha) \frac{u_{B}}{2}
$$

where $b$ is a function such that $b(0)=0$ and $\lim _{\alpha \rightarrow \infty} b(\alpha)=1$ which branches heuristically

both pressure regimes and $\alpha=\frac{4 \lambda_{i}^{2}}{l_{x} l_{y}}$ is inversely proportional to the pressure squared. As shown in Figures 7, 8 and Figure 11 in the Appendix, good agreements with PIC simulation results were found using a quite steep function:

$$
b(\alpha)=\tanh ^{0.2}(\alpha)
$$

This function yielded satisfactory results for argon but another form of function might be more suitable for other types of gases. In the next section, it is shown that the 1D heuristic formula can be straightforwardly extended to the 2D case.

\subsection{A corrected heuristic formula}

The 1D heuristic formula proposed by [12] may be easily corrected to take into account first order $2 \mathrm{D}$ effects due to the density profile in the direction perpendicular to the ion velocity vector. As shown in subsection 2.3, this correction factor is $2 / \pi$ at high pressure. Since the mean normalized density $\left(\beta_{x, y}\right)$ depends weakly on the pressure and that it has no simple expression, we may keep the value $2 / \pi$ for all pressure regimes. The corrected heuristic formula then becomes

$$
\begin{aligned}
& h_{L, \text { heur }, x}=0.55\left[3+0.5 \frac{l_{y}}{\lambda_{i}}+0.2 \frac{T_{i}}{T_{e}}\left(\frac{l_{y}}{\lambda_{i}}\right)^{2}\right]^{-1 / 2} \\
& h_{L, \text { heur }, y}=0.55\left[3+0.5 \frac{l_{x}}{\lambda_{i}}+0.2 \frac{T_{i}}{T_{e}}\left(\frac{l_{x}}{\lambda_{i}}\right)^{2}\right]^{-1 / 2}
\end{aligned}
$$


where $0.55 \approx(2 / \pi) \times 0.86$. It should be noted that $h_{L, \text { heur }, x}-$ which is the $h$ factor relative to the flux of ions collected on $y= \pm l_{y} / 2-$ depends on $l_{y}$, and not $l_{x}$. The global heuristic $h$ factor is then

$$
h_{2 D, \text { heur }}=\frac{l_{x} h_{L, \text { heur }, x}+l_{y} h_{L, \text { heur }, y}}{l_{x}+l_{y}}
$$

Figures 7 and 8 show comparisons between the PIC simulations, the two versions of the fluid theory (with different expressions of the ion-neutral collision frequency) and the corrected heuristic formula. The solid dots of Figure 7 are PIC values when varying the pressure between 0.3 mTorr and $100 \mathrm{mTorr}$. Figure 8 corresponds to several values of $l_{x}$, from $7.5 \mathrm{~mm}$ to $75 \mathrm{~mm}$ implemented in the PIC simulation, while $l_{y}$ was always kept to $3 \mathrm{~cm}$. The corrected heuristic formula shows a very good agreement with PIC simulations for both parametric studies because it accounts well for the intermediate pressure regime. The prediction of the original $2 \mathrm{D}$ fluid theory described in section 2 agrees reasonably well with the PIC simulation results at low pressure but the discrepancy becomes significant in the intermediate pressure regime. The prediction of the variation of $h$ factor with the aspect ratio is also quite poor with the original theory. However, the agreement is greatly improved when the modified ion-neutral collision frequency (Equations 53 and 54) is introduced in the fluid theory.

Before we conclude this comparison section, we note that at the lowest pressures, it was found in the PIC simulation that the $h$ factor tends to slightly decrease. For these very low pressures, the electron distribution function predicted by PIC simulation becomes strongly anisotropic. This may well be the case in a real system but it could also be an artefact of the heating method implemented in the PIC simulation.

\section{Conclusion and further discussions}

The so-called $h$ factors were investigated in 2D Cartesian discharges using a fluid theory and the verification by PIC simulations. The separated roles of pressure and aspect ratio were carefully investigated. The theory predicts the electron temperature with a high degree of accuracy. When the assumption of constant ion-neutral collision frequencies is made, the theory fails in the intermediate pressure regime and the prediction of $h$ factors lacks of accuracy for pressures higher than a few mTorr and below hundreds of mTorr. The theory is greatly improved when a pressure-dependent velocity is used in the ionneutral collision frequency. A corrected heuristic formula, based on the widely used 1D formula, was proposed and it was shown that it matches very well the PIC simulation results in a wide range of pressure and for several aspect ratios of the system. This formula (Equation 55, together with Equations 56, and 57) should be used for global models of a 2D Cartesian discharge. The reasoning developed in this paper applies to cylindrical geometries (see the work of Zhang [15]) and to 3D discharges as well, but with different numerical coefficients.

The PIC simulations have also shown interesting properties on non-ambipolarity.

Figure 9 shows electron and ion currents collected on one wall of a square box. The 


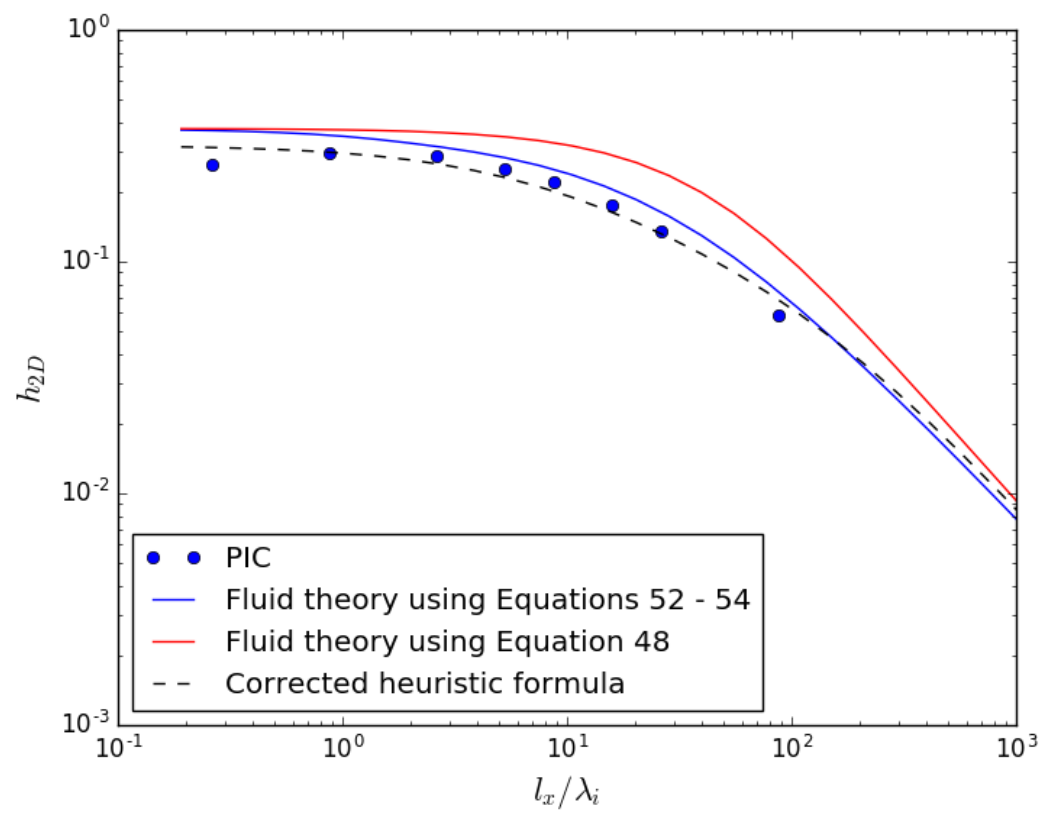

Figure 7: Predicted $h_{2 D}$ factors compared with PIC simulation results for various values of pressure. $l_{x}=l_{y}=3 \mathrm{~cm}, p$ from $0.2 \mathrm{mTorr}$ to 1.0 Torr. The two versions of the fluid model using different assumptions for the ion-neutral collision frequency are plotted together with the corrected heuristic formula (Equations 55 - 57).

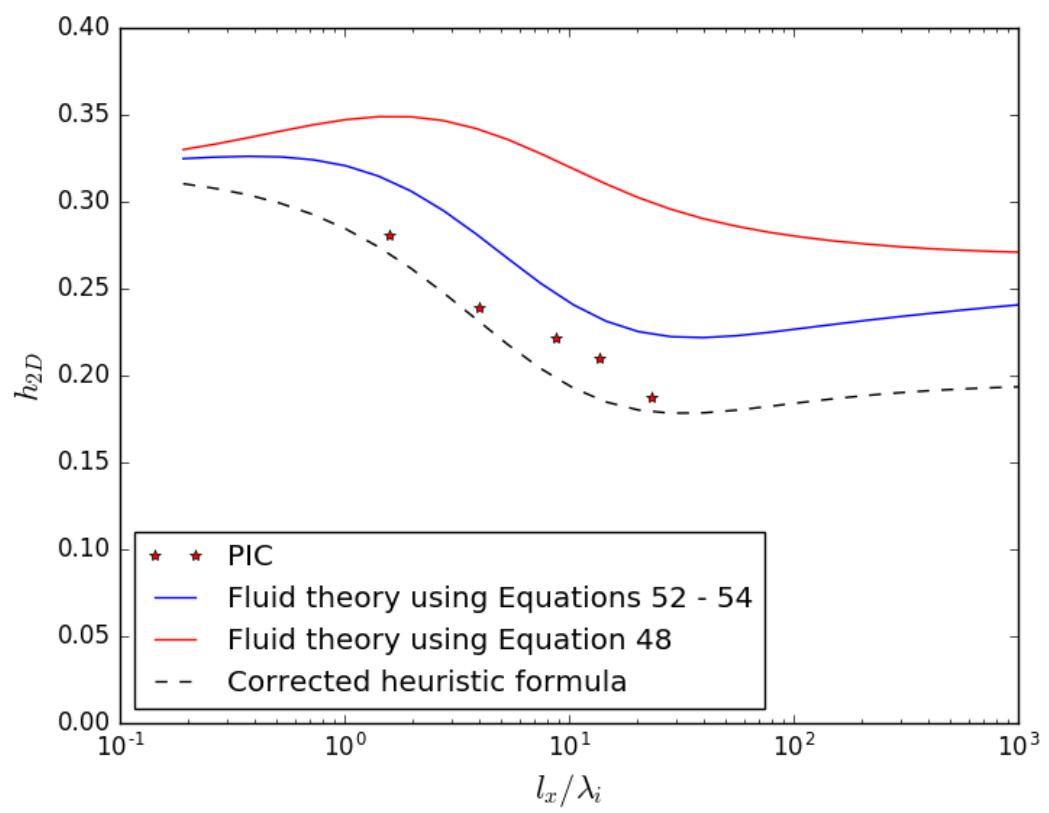

Figure 8: Predicted $h_{2 D}$ factors compared with PIC simulation results for various dimensions in $(O x)$ direction. $l_{y}=3.0 \mathrm{~cm}, p=10 \mathrm{mTorr}, l_{x}$ from $0.6 \mathrm{~mm}$ to $6 \mathrm{~m}$. The two versions of the fluid model using different assumptions for the ion-neutral collision frequency are plotted together with the corrected heuristic formula (Equations 55- 57). 


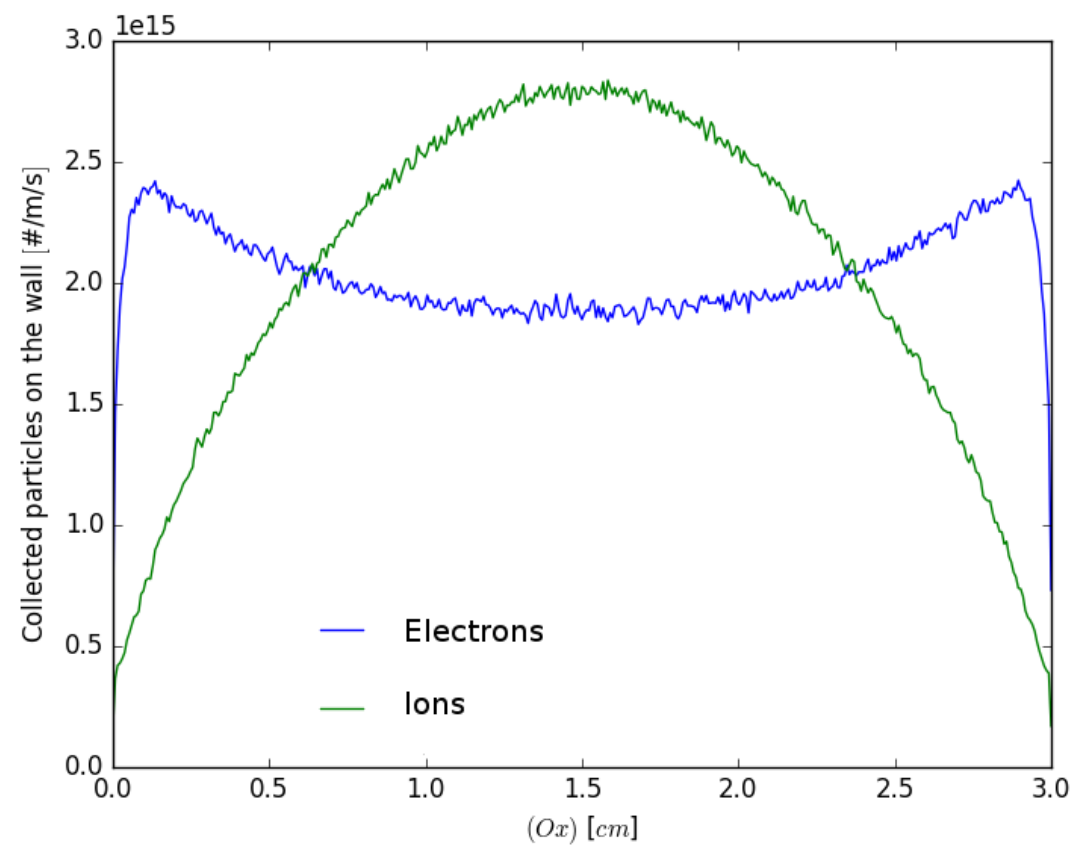

Figure 9: Electron and ion current densities in steady-state at $10 \mathrm{~m}$ Torr and $19 \mathrm{~kW} / \mathrm{m}^{3}$.

average electron and ion currents are equal, which means that the global flux balance is satisfied, as it must. However, while the ion flux profile follows that of the plasma density, the electron flux features a minimum at the center of the wall and maxima near the sheath edges. PIC simulations carried out here are consistent with a discharge chamber with conductive walls and shows that large currents should be flowing through the walls. Moreover, when the dimensions of the discharge chamber in $(O x)$ and $(O y)$ directions are not equal, there are more ions collected on the longer edge and more electrons on the shorter edge, while global current balance is respected. These results are confirming the results found by T. Lafleur [23]. It highlights the fact that only global $h$ factors should be used in global models, especially for electron power and particle balance. It would be of high interest to conduct these parametric studies again with dielectric walls to see how the profiles of Figure 9 are modified. The heating mechanism could also be improved, for example by solving Maxwell's equations [24], in order to better describe very low pressure regimes with the PIC simulations.

Finally, direct measurements of the $h$ factors remain a challenge but would be of fundamental importance to validate the theory presented in this paper.

\section{Acknowledgments}

The authors are grateful to A. Tavant and R. Martorelli for the numerous helpful discussions, as well as to A. Lichtenberg and M. Lieberman for their reviews of the manuscript. This work was granted access to the HPC resources of [TGCC/CINES/IDRIS] under the allocation 2017-A0020510092 made by GENCI, and it 
was partially funded by CHEOPS project that has received funding from the European Union's Horizon 2020 research and innovation programme under grant agreement No 730135.

\section{Appendix}

Derivation of the model equations

Momentum conservation equation in the $(O x)$ direction yields:

$$
u_{x}^{\prime}+\frac{u_{B}^{2}}{u_{x}^{2}}\left(\nu_{x}-u_{x}^{\prime}\right)+\nu_{i z}+\nu_{i}=0 \Leftrightarrow \frac{u_{x}^{2}-u_{B}^{2}}{\nu_{x} u_{B}^{2}+\left(\nu_{i z}+\nu_{i}\right) u_{x}^{2}} d u_{x}=-d x
$$

Introducing the variable $s=\frac{u_{x}}{u_{B} \sqrt{\beta}}$, with $\beta=\frac{\nu_{x}}{\nu_{i z}+\nu_{i}}$, and taking into account the fact that $s=0$ when $x=0$, this leads after integration to:

$$
\beta s-(\beta+1) \arctan (s)=-\sqrt{\nu_{x}\left(\nu_{i z}+\nu_{i}\right)} \frac{x}{u_{B}}
$$

Replacing with $u_{x}$, the velocity field is implicitly given by Equation 18 .

Since $u_{x}^{\prime}=\frac{u_{B}^{2} \nu_{x}+u_{x}^{2}\left(\nu_{i z}+\nu_{i}\right)}{u_{B}^{2}-u_{x}^{2}}$ and $f^{\prime} / f=\left(\nu_{x}-u_{x}^{\prime}\right) / u_{x}$, we derive:

$$
\frac{d}{d x}(\ln f)=\frac{f^{\prime}}{f}=-\frac{\nu_{x}+\nu_{i z}+\nu_{i}}{u_{B}^{2}-u_{x}^{2}} u_{x}
$$

Hence,

$$
\begin{aligned}
f(x) & =\exp \left[-\left(\nu_{i z}+\nu_{i}+\nu_{x}\right) \int_{0}^{x} \frac{u_{x}(X)}{u_{B}^{2}-u_{x}(X)^{2}} d X\right] \\
& =\exp \left[-\left(\nu_{i z}+\nu_{i}+\nu_{x}\right) \int_{0}^{u_{x}(x)} \frac{v d v}{u_{B}^{2} \nu_{x}+v^{2}\left(\nu_{i z}+\nu_{i}\right)}\right]
\end{aligned}
$$

Which leads to the density profile of Equation 22 .

\section{Solutions of the model}

$\nu_{x}$ and $\nu_{y}$ coefficients represent the importance of ionization in $(O x)$ and $(O y)$ directions. We attempted to figure out how the ratio $\nu_{x} / \nu_{y}$ varies with $l_{x} / l_{y}$. Numerical solutions of the model were computed for many values of pressure and aspect ratios and plotted in Figure 10. An approximate equation was found under the form:

$$
\left(\frac{\nu_{y}}{\nu_{x}}\right)=\tan ^{2} \theta=\left(\frac{l_{x}}{l_{y}}\right)^{\alpha}
$$

$\alpha$ is a power coefficient that varies between 1.2 and 2, that depends weakly on the pressure and on the dimensions of the system. In the high pressure limit, Equation 34 


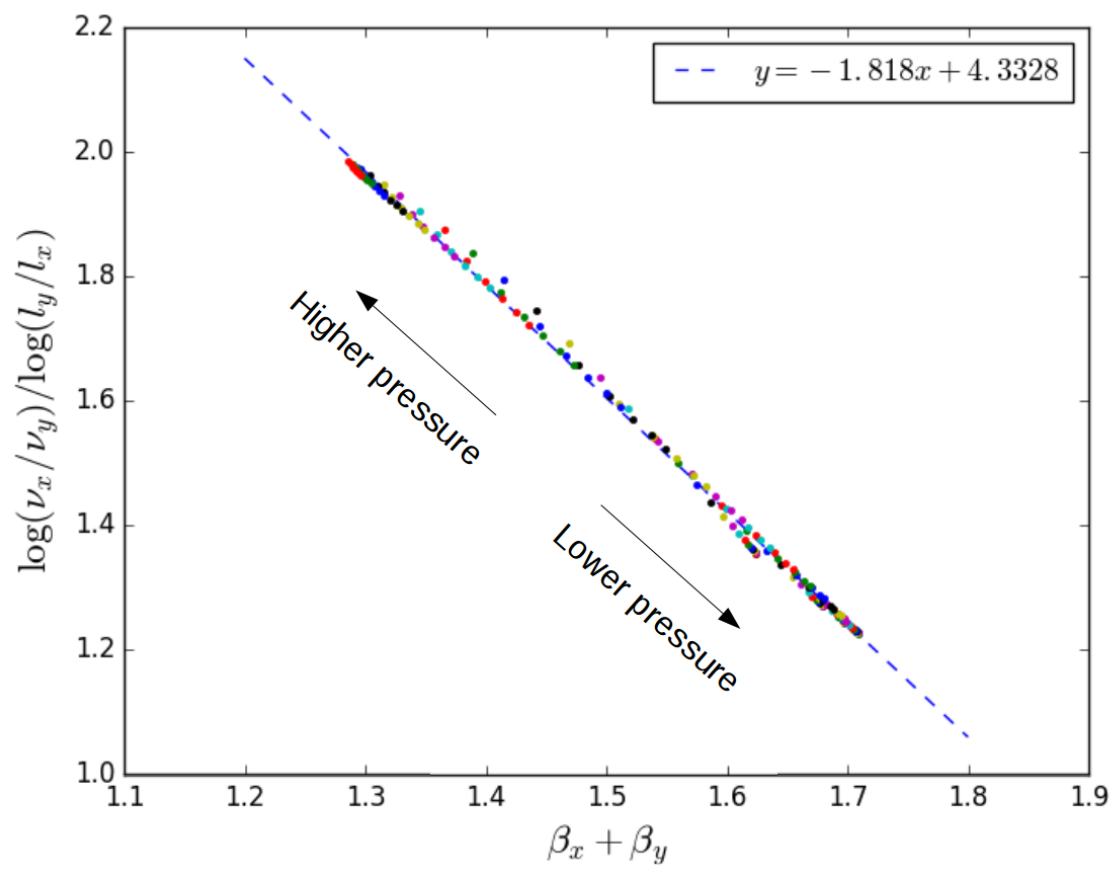

Figure 10: Numerical resolution of the model equations with $\nu_{i}=\bar{v}_{i} / \lambda_{i}$, for pressures between $0.2 \mathrm{~m}$ Torr and $160 \mathrm{mTorr}, l_{x}$ between $0.15 \mathrm{~cm}$ and $3 \mathrm{~cm}$, and $l_{y}=3 \mathrm{~cm}$.

shows that this coefficient is equal to 2. The variation of $\alpha$ follows the same trend as the sum of the mean normalized density profiles $\beta_{x}+\beta_{y}$.

$$
\alpha=2-1.8\left(\beta_{x}+\beta_{y}-\frac{4}{\pi}\right)
$$

Coefficient 1.8 is found from the fit of Figure 10. The value of this coefficient does not depend on the type of law for the ion-neutral collision rate (function $b$ in Equation 53 does not affect the value of this coefficient, as long as it verifies $b(0)=0$ and $\left.\lim _{\alpha \rightarrow \infty} b(\alpha)=1\right)$. The slope of 1.8 is almost not affected when the characteristics of the gas such as the mean cross section for ion-neutral collisions and the ionization reaction rate are changed.

\section{Predicted $h$ factors for one side of the discharge chamber}

The advantage of focusing on only one wall of the discharge chamber is to compare the relative role of the pressure and the size of the system in the direction perpendicular to the investigated wall. According to Equation 3 , the pre-sheath drop for one side should depend only on the ratio $l / \lambda_{i}$. Figure 11 , where both parametric studies conducted in this study were plotted together, illustrates that the model developed here (with pressure dependant ion reference velocity, cf Equations 53 and 54 accounts for these distinct trends reasonably well. However, the corrected heuristic formula (Equations 55 and 56) still shows a good agreement with both parametric studies. 


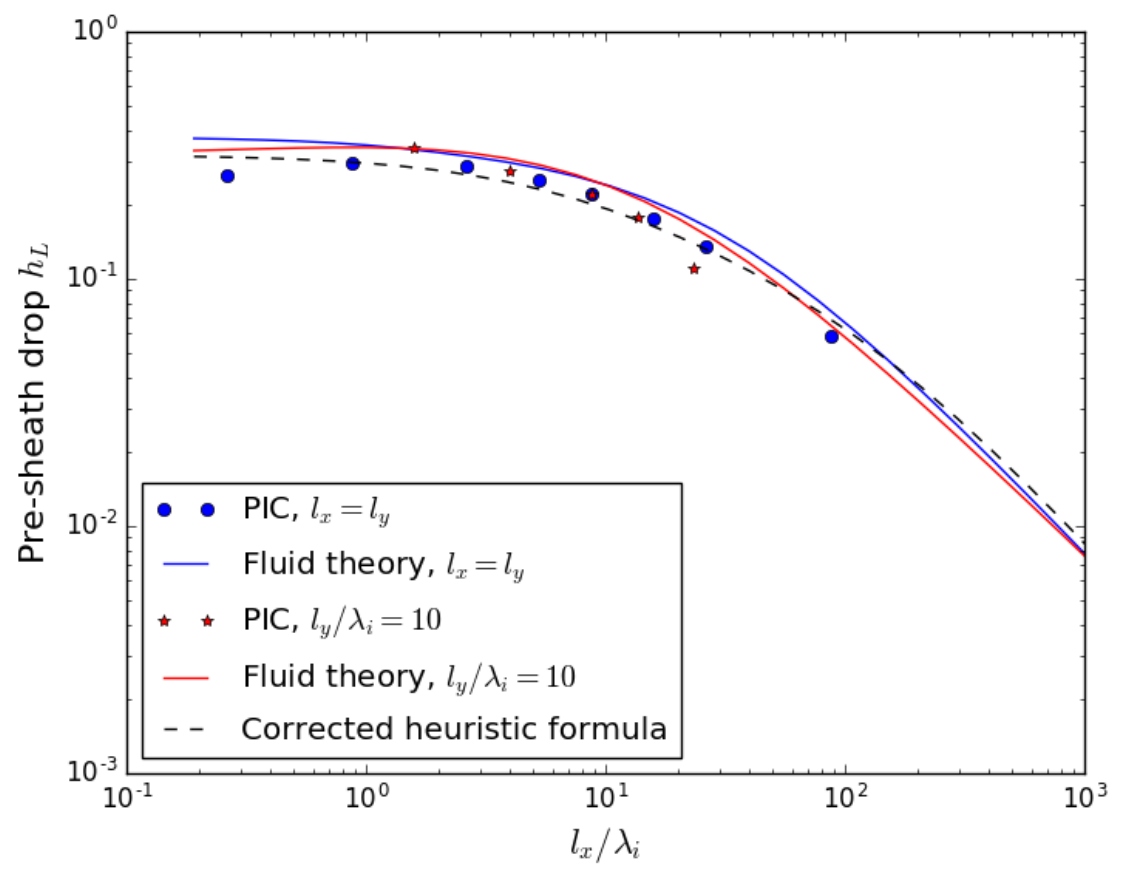

Figure 11: Predicted $h$ factors for ion collection on the side wall $x=l_{x} / 2$ using the model solution with pressure-dependant reference velocity for the ions (Equations 53 and 54, plotted together with the corrected heuristic formula, and PIC simulation results. 


\section{References}

[1] D. M. Goebel and I. Katz. Fundamentals of Electric Propulsion: Ion and Hall Thrusters. WileyInterscience, wiley edition, 2008.

[2] P. Grondein, T. Lafleur, P. Chabert, and A. Aanesland. Global model of an iodine gridded plasma thruster. Physics of Plasmas, 23(033514), 2016.

[3] P. Chabert, J. Arancibia Monreal, J. Bredin, L. Popelier, and A. Aanesland. Global model of a gridded-ion thruster powered by a radiofrequency inductive coil. Physics of Plasmas, 19(7), 2012.

[4] M. A. Lieberman and A. J. Lichtenberg. Principles of Plasma Discharges and Materials Processing. John Wiley and Sons, second edition, 2005.

[5] P. Chabert and N. Braithwaite. Radio-Frequency Plasmas. Cambridge University Press, 2011.

[6] A. Perret, P. Chabert, J. Jolly, and J.-P. Booth. Appl. Phys. Lett., 86(1):021501, 2005.

[7] T. Lafleur and P. Chabert. Edge-to-center density ratios in low-temperature plasmas. Plasma Sources Sci. Technol., 24(025017):3, 2015.

[8] W. Schottky. Diffusionstheorie der positiven Säule. Phys. Zeits, 25(635), 1924.

[9] L. Tonks and I. Langmuir. A General Theory of the Plasma of an Arc. Phys. Rev., 34(876), 1929.

[10] V. A. Godyak. Soviet Radio Frequency Discharge Research. Delphic ass. inc., 1986.

[11] J.-L. Raimbault and P. Chabert. Edge-to-center plasma density ratio in high density plasma sources. Plasma Sources Sci. Technol., 18(014017), 2009.

[12] C. Lee and M. A. Lieberman. Global model of $\mathrm{Ar}, \mathrm{O}_{2}, \mathrm{Cl}_{2}$, and $\mathrm{Ar} / \mathrm{O}_{2}$ high-density plasma discharges. Journal of Vaccum Science and Technology, 13(0734-2101), 1995.

[13] G.-H. Kim, H.-C. Lee, and C.-W. Chung. Experimental investigation of edge-to-center density ratio in inductively coupled plasmas. Physics of Plasmas, 17(7):073504, July 2010.

[14] G.S. Kino and E. K. Shaw. Two-Dimensional Low-Pressure Discharge Theory. The Physics of Fluids, 9(587), 1966.

[15] Y. Zhang, C. Charles, and R. Boswell. Principle of radial transport in low temperature annular plasmas. Physics of Plasmas, 22(073510), 2015.

[16] Lucken, R. and Croes, V. and Lafleur, T. and Raimbault, J.-L. and Bourdon, A. and Chabert, P. Global models of plasma thrusters: Insights from PIC simulation and fluid theory. volume 2017-323. International Electric Porpulsion Conference, 2017.

[17] V. Croes, T. Lafleur, Z. Bonaventura, A. Bourdon, and P. Chabert. 2D Particle-In-Cell simulations of the electron drift instability and associated anomalous electron transport in Hall-Effect Thrusters. Plasma Sources Sci. Technol., 26(034001), 2017.

[18] A. V. Phelps. The application of scattering cross sections to ion flux models in discharge sheaths. Journal of Applied Physics, 76(2):747, June 1998.

[19] S. F. Biagi. Programm magboltz v7.1. cross section compilation. see www.lxcat.net. retrieved on November 16, 2016, 2004.

[20] C. K. Birdsall and A. B. Langdon. Plasma Physics via Computer Simulation. McGraw-Hill, Inc., 1991.

[21] M. M. Turner, A. Derzsi, Z. Donko, D. Eremin, S. J. Kelly, and T. Mussenbrock. Simulation benchmarks for low-pressure plasmas : capacitive discharges. Plasma Physics, 20(1):013507, 2012.

[22] N. Sternberg and V. Godyak. Two-dimensional cylindrical plasma for intermediate gas pressures. Plasma Sources Sci. Technol., 20(1):015018, 2011.

[23] T. Lafleur and R. W. Boswell. Particle-in-cell simulations of ambipolar and nonambipolar diffusion in magnetized plasmas. Physics of Plasmas, 19(053505):4, 2012.

[24] J.P. Verboncoeur, A.B. Langdon, and N.T. Gladd. An object-oriented electromagnetic PIC code. Computer Physics Communication, 87(199-211), 1995. 